\title{
Formas digestivas da doença de Chagas e carcinogênese: um estudo de associação
}

\section{Digestive forms of Chagas disease and carcinogenesis: a study of association}

\author{
Calo Eduardo Gullo"; Cássia Fernanda Estofolete ${ }^{1}$; Cristiane Damas Gil²; Adriana Borgonovi Christiano, ACbC - Sp3; \\ JoÃo Gomes Netinho, TCBC - SP4
}

\author{
RE S U M O
}

\begin{abstract}
Os autores analisam a relação entre carcinogênese gastrintestinal e doença de Chagas, com base em revisão pormenorizada da literatura. Para tal, foram selecionados estudos epidemiológicos, experimentais e de descrição anatomopatológica com material humano. O artigo discute a possibilidade de a proteção ser conferida por fatores celulares morfocinéticos, imunológicos e neuroendócrinos não totalmente conhecidos e que seriam secundários à degeneração plexular. Também são apresentados aspectos relacionados à interação parasito-hospedeiro, sob o ponto de vista da modulação epitelial da mucosa colônica, e suas implicações antitumorais. Por fim, expõe-se o mecanismo fisiopatológico de desenvolvimento da neoplasia de esôfago em pacientes com megaesôfago. Conclui-se que a colopatia chagásica, especialmente o dano neuronal intrínseco, constitui modelo de estudo que pode contribuir no entendimento da carcinogênese colorretal.
\end{abstract}

Descritores: Doença de Chagas. Denervação autônoma. Neoplasias colorretais. Megaesôfago. Neoplasias esofágicas.

\section{INTRODUÇÃO}

O risco de desenvolvimento de carcinoma epidermóide de esôfago nos pacientes portadores de megaesôfago é 33 vezes superior ao da população em geral' ${ }^{1}$. Em relação ao megacólon chagásico, embora em teoria tenha todas as características para ser enquadrado como um potencial e importante fator de risco para o carcinoma do cólon, um estudo experimental aponta que o megacolon chagásico parece conferir efeito protetor na carcinogênese colorretal ${ }^{2}$. A revisão da literatura indica apenas oito casos desta associação e, analisadas em conjunto as séries de levantamentos de câncer em megas chagásicos, encontrase incidência de 4,8\% em megaesôfago e 0,1\% em megacólon ${ }^{3}$.

Pesquisadores relataram uma ocorrência menor de tumores de cólon quimicamente induzidos em ratos chagásicos com ou sem megacólon, na fase crônica da infecção ${ }^{4}$. Garcia et al. descreveram uma frequência reduzida de pólipos intestinais em pacientes com megacólon chagásico ${ }^{5}$, cujo aparecimento constitui, tradicionalmente, etapa inicial no desenvolvimento de adenocarcinomas do cólon. Uma hipótese que tenta explicar o fenômeno é que a denervação plexular mioentérica devido à infecção pelo T. cruzi tornaria a mucosa do cólon menos responsiva à ação de carcinógenos químicos, conferindo, então, maior resistência ao desenvolvimento de tumores. Seu papel anticarcinogênico também é considerado em outras etiologias, conforme demonstrado por Polli-Lopes et al. em ratos submetidos à denervação mioentérica pelo cloreto de benzalcônio (CBA). Nesses animais, houve redução significante do tamanho e do número de tumores gástricos induzidos pelo carcinógeno N-metil-N'-nitro-Nnitrosoguanidina (MNNG) ${ }^{6}$, corroborando as evidências de que a denervação mioentérica constitui fator independente de proteção contra a carcinogênese gastrintestinal.

Dessa maneira, as alterações que ocorrem na infecção crônica pelo T. cruzi, em especial a denervação mioentérica, responsável pelas formas digestivas da doença, guardam estreita relação com a etiopatogenia da carcinogênese esofágica e colorretal. Considerando tais aspectos, serão abordados os mecanismos fisiopatológicos suscitados pelo parasita e seus efeitos, ora positivos, ora negativos, sobre as neoplasias do trato digestório, cujo entendimento é de peculiar interesse dada a importância que as mesmas assumem na atualidade.

\section{MÉTODOS}

Neste estudo utilizou-se a revisão de literatura, com pesquisa em conteúdo específico percorrendo as

Trabalho realizado no Hospital de Base - Faculdade de Medicina de São José do Rio Preto- FAMERP - São José do Rio Preto - SP-BR. 1. Médicos da Faculdade de Medicina de São José do Rio Preto- FAMERP - São José do Rio Preto - SP-BR; 2. Professora Adjunta da Universidade Federal de São Paulo - São Paulo - SP-BR; 3. Coloproctologista do Hospital de Base- FAMERP - São José do Rio Preto - SP-BR; 4. Chefe da Disciplina de Coloproctologia Hospital de Base / FAMERP - São José do Rio Preto - SP-BR. 
seguintes fases: identificação do tema, categorização dos assuntos, interpretação dos resultados e síntese do conhecimento evidenciado nos artigos analisados. As referências bibliográficas foram levantadas em livros e em artigos encontrados nas bases de dados Literatura LatinoAmericana e do Caribe em Ciências da Saúde (LILACS) e MEDLINE. Os descritores utilizados foram: doença de Chagas (Chagas disease), denervação autônoma (autonomic denervation), neoplasias colorretais (colorectal neoplasms), megaesôfago (megaesophagus) e neoplasias esofágicas (esophageal neoplasms). Após, o material obtido foi analisado segundo objetivo proposto, metodologia empregada e resultados obtidos, bem como discussão e considerações.

\section{RESULTADOS}

\section{Sistema nervoso entérico e etiopatogenia da colopatia chagásica}

O trato gastrintestinal é relacionado pela parte autônoma do sistema nervoso, por meio das inervações extrínseca e intrínseca. As divisões simpática e parassimpática suprem a inervação extrínseca atuando diretamente nos órgãos-alvo ou, indiretamente, modulando a atividade da inervação intrínseca ${ }^{7,8}$. Esta, por sua vez, é efetuada pelo Sistema Nervoso Entérico (SNE), uma coleção de neurônios e células de suporte ${ }^{9}$. Enquanto as fibras nervosas simpáticas e parassimpáticas projetam-se para a parte central do sistema nervoso via gânglios da raiz dorsal e da medula espinal, respectivamente, o SNE pode trabalhar independente do mesmo, sendo por isso considerado o cérebro do intestino. Localizada na parede do trato gastrintestinal, esta extensa e difusa rede de $10^{8}$ neurônios sensoriais, interneurônios e neurônios motores controla, principalmente, a peristalse, as mudanças no fluxo sanguíneo local e a secreção de água e de eletrólitos ${ }^{10}$.

Sabe-se que o envolvimento do SNE é crucial na instalação das desordens gastrintestinais que ocorrem na doença de Chagas, de modo que anormalidades importantes são observadas em vários componentes do mesmo. A degeneração plexular ou denervação pósganglionar parassimpática intrínseca causa hipertrofia muscular e hiperplasia da mucosa, ambas responsáveis pelas visceromegalias (megaesôfago e megacólon) na fase crônica da infecção pelo $T$. cruzl². Entretanto, alterações secundárias ao dano neuronal, envolvendo elementos moleculares, celulares e extracelulares, ocorrem independentemente da dilatação dos órgãos-alvo.

O mecanismo fisiopatológico pelo qual a destruição de neurônios mioentéricos se processa, bem como suas repercussões locais e sistêmicas, permanece discutível ${ }^{11}$. Várias hipóteses foram criadas e estudos recentes consideram que o T. cruzi pode causar lesão tissular tanto por meio de sua ação direta em células-alvo infectadas quanto indiretamente, pela indução do desenvolvimento de hipersensibilidade e participação de fenômenos autoimunes $^{12}$. De fato, um antígeno flagelar do parasita mimetiza uma proteína expressa pelos neurônios supracitados, resultando em imunorreatividade cruzada que atrai células imunológicas para dentro dos gânglios e causa uma ganglionite mioentérica aguda ${ }^{13}$.

Histopatologicamente observa-se uma diminuição dos neurônios mioentéricos intrínsecos e das células intersticiais de Cajal nos segmentos acometidos do esôfago e do cólon ${ }^{13}$. Como consequência, a musculatura lisa desses órgãos passa a responder com contrações desordenadas e de intensidade variável, tanto hiperreativas quanto hiporreativas, dependendo do estímulo feito ${ }^{14}$. O distúrbio motor originado no esôfago leva ao aparecimento de sintomas típicos como disfagia, regurgitação e emagrecimento que pode ocorrer após vários anos do diagnóstico sorológico e/ou do estabelecimento da lesão neuromotora ${ }^{14}$.

O megacólon, por sua vez, cursa com constipação crônica na qual a pressão das fezes desidratadas e endurecida que ficaram retidas produz ulcerações extensas na mucosa colônica². Fibrose, hiperplasia da mucosa e inflamação com predomínio de células linfomononucleares também são frequentemente encontradas em exames anatomopatológicos de peças cirúrgicas ou de autópsias de pacientes com megacólon chagásico ${ }^{5}$.

\section{Formas digestivas da doença de Chagas e tumorigênese}

\subsection{Repercussões da denervação mioentérica na mucosa colônica}

\subsubsection{Epitélio}

A denervação do cólon, em ratos, diminui o número de focos de cripta intestinal aberrante, a sua taxa de divisão e o acúmulo de ß-catenina, proteína dita como "facilitadora" da carcinogênese colônica ${ }^{15}$. A proliferação celular após uma única injeção do carcinógeno dimetilhidrazina $(\mathrm{DMH})$ foi intensamente inibida nestes animais. Demonstrou-se que a alteração de qualquer um de tais parâmetros deve influenciar ativamente o processo de carcinogênese, e que os mesmos estão interrelacionados. O aumento da ß-catenina parece levar à desregulação mitótica das criptas, fenômeno crucial para a formação das unidades criptais aberrantes, sendo que ambos são dependentes da proliferação celular epitelial e mostraram intensa diminuição em animais denervados ${ }^{15}$.

A inibição da carcinogênese colorretal que se verifica na doença de Chagas também parece envolver proteínas metalotioneínas (MT), proteínas de baixo peso molecular, que podem executar várias funções como a detoxificação e clearance de radicais livres de tecidos e células. As metalotioneínas podem exercer funções protetoras durante o dano ao DNA, presumivelmente por 
atuarem como antioxidantes. Observou-se que a carcinogênese colorretal é caracterizada por uma diminuição significativa da expressão de $\mathrm{MT}^{16}$. A infecção pelo T. cruzi, por sua vez, causa um aumento pronunciado da expressão de MT, assim como ocorre na utilização de anti-inflamatórios não hormonais (AINH). Escalante et al. sugerem que o aumento da formação de criptas colônicas super-expressando MT deva estar relacionada à proteção contra carcinogênese promovida pelo $T$. cruzi e $\mathrm{AINH}^{16}$. A relevância oncológica destas criptas é ainda incerta, mas seu surgimento deve prevenir mutações adicionais que poderiam eventualmente levar ao fenótipo neoplásico, além de constituírem biomarcadores de melhor prognóstico no câncer colorretal. O estudo de seu potencial metastático demonstra uma variação clonal da resposta ao cádmio em linhas de células tumorais humanas, a qual deve estar relacionada ao nível de expressão de $\mathrm{MT}^{16}$.

\subsubsection{Células imunológicas}

Vários tipos celulares, incluindo neutrófilos, linfócitos, macrófagos, células dendríticas e mastócitos estão presentes na mucosa entérica e na musculatura lisa. Cada um desses tipos celulares podem ser encontrados em íntima associação com elementos do SNE, fibras nervosas vagais e nervos sensoriais espinais ${ }^{10}$ e cooperam com o SNE estabelecendo a primeira linha de defesa contra a invasão de antígenos. Todas as células imunológicas são fontes potentes de sinais parácrinos para o SNE, a exemplo dos mastócitos, que armazenam mediadores farmacológicos em seus grânulos implicados na resposta a estímulos imunológicos potencialmente prejudiciais à integridade do trato gastrintestinal, incluindo proteínas com atividades antitumorais ${ }^{17}$

Na colopatia chagásica, diversas alterações nesses elementos são encontradas, incluindo infiltração de linfócitos $T$ e aumento do número de mastócitos ${ }^{13}$, provavelmente relacionadas à denervação colônica per se. A associação entre processos inflamatórios crônicos e carcinogênese tem sido muito aventada ${ }^{18}$, particularmente envolvendo gastrite, pancreatite crônica e retocolite ulcerativa no desenvolvimento de neoplasias de estômago, pâncreas e cólon, respectivamente ${ }^{19,20,21}$.

Entretanto, apesar do intenso processo inflamatório observado nos segmentos acometidos do cólon na vigência da infecção pelo $T$. cruzi, a influência da denervação plexular sobre o sistema imunológico entérico parece contradizer a assertiva acima. Estofolete et al. demonstraram em estômagos denervados que as neoplasias epiteliais gástricas induzidas por MNNG também apresentam um aumento significante do número de mastócitos, porém com uma mudança fenotípica de "mastócitos de mucosa" para "mastócitos de tecido conectivo", os quais parecem exibir maior liberação de mediadores antitumorais ${ }^{17}$. Estudos adicionais devem ser desenvolvidos para verificar se há correspondência histológica em cólons denervados, bem como determinar o status funcional de elementos celulares e moleculares na imunomodulação do câncer nesses órgãos.

\subsubsection{Sistema neuroendócrino}

É conhecido que a lesão neuronal mioentérica altera de maneira significante o sistema neuroendócrino gastrintestinal, e que a acetilcolina, a serotonina, a noradrenalina, a somatostatina e o polipeptídeo intestinal vasoativo (VIP) podem produzir modificações celulares morfocinéticas ${ }^{15}$. Por exemplo, o VIP parece aumentar o número de tumores induzidos pelo carcinógeno DMH em ratos. Interessantemente, também foi observado que as junções aderentes entre as células epiteliais são reguladas pela gastrina, cujos níveis são influenciados pela denervação gástrica ${ }^{15}$. Especula-se a possibilidade de que a degeneração plexular colônica também altere as concentrações deste hormônio, e assim o controle da adesão entre células epiteliais vizinhas, um fato que pode contribuir no entendimento da carcinogênese colorretal ${ }^{15}$.

Ainda, fatores intraluminares, tais como a flora bacteriana e o pH, e parietais, relacionados às modificações nos componentes da parede intestinal como consequência da visceromegalia, poderiam influenciar os níveis de neurotrasmissores, neuropeptídeos e outros ainda desconhecidos. Por fim, alterações nas atividades da enzima óxido nítrico sintase foram demonstradas no intestino de camundongos infectados pelo T. cruzß . Entretanto, Garcia et al. relataram que o óxido nítrico é suscitado pela resposta inflamatória contra a infecção colônica e seria o responsável pela destruição dos neurônios nos plexos intestinais ${ }^{5}$; assim, o aumento de sua expressão constituiria causa, e não consequência, do megacólon.

\subsection{Megaesôfago e câncer}

De maneira inversa à colopatia chagásica, portadores de megaesôfago apresentam, reconhecidamente, maior propensão para o desenvolvimento de carcinoma espinocelular de esôfago, o qual pode se localizar em qualquer porção do órgão nesses pacientes e cuja prevalência (3,9\% a 10\%) determina implicações práticas de seguimento endoscópico ${ }^{22,23}$. O adenocarcinoma do esôfago também pode ser observado em pacientes submetidos a dilatação do cárdia ou miotomia da transição esofagogástrica, pois tais intervenções predispõem ao refluxo ácido e, então, metaplasia intestinal (Barrett), lesão precursora desse tipo de tumor ${ }^{23}$.

A simples infecção pelo T. cruzi não justifica tal associação, uma vez que não há aumento na frequência de câncer de esôfago em pacientes chagásicos sem megaesôfago ${ }^{3}$. Por outro lado, a presença de megaesôfago confere o mesmo risco de malignização daquele apresentado por indivíduos com acalasia idiopática. Assim, sugere-se que a relação do câncer esofágico, como na acalasia, é com o megaesôfago, e não com a doença de Chagas $^{22}$. 
Este achado pode ser explicado pela estase alimentar consequente à dilatação do órgão, levando à esofagite crônica, deficiências vitamínicas associadas à subnutrição e contato prolongado entre os agentes carcinogênicos da dieta e a mucosa ${ }^{3,23}$. A ação carcinogênica pode estar relacionada à produção, a partir de nitratos da dieta e mediada por bactérias presentes no líquido de estase, de compostos N-nitrosos na luz do órgão, cuja capacidade mutagênica no DNA celular já foi demonstrada em estudos experimentais ${ }^{1}$. Assim, o supercrescimento bacteriano constituiu outro possível fator envolvido no aparecimento de displasia epitelial e câncer esofágico.

\section{CONSIDERAÇÕES FINAIS}

Mostrou-se que há uma associação positiva entre a doença de Chagas e o leiomioma uterino ${ }^{5}$, este fato evidencia que a relação entre a infecção pelo $T$.cruzi e a incidência de neoplasias mostra-se muito complexa. Sabese que o $T$. cruzi produz um processo de inflamação crônica persistente ao longo do tubo digestório com distribuição desigual. Algumas infecções crônicas por vírus, bactérias e parasitas são bem conhecidas como fatores de risco em cânceres humanos. Resta saber por que a infecção crônica pelo T.cruzi não se comporta da mesma maneira.

As infecções crônicas causadas por parasitas intracelulares, entre eles os protozoários Toxoplasma gondii e o Besnoitia jellison ${ }^{24}$ podem induzir determinado grau de proteção contra alguns tipos de tumores ${ }^{24}$. Um papel alternativo da própria infecção contra a carcinogênese seria o aumento da resistência imunológica decorrente da inflamação crônica, otimizando também a capacidade de vigilância dos sistemas de defesa do organismo em relação ao desenvolvimento de células mutantes ${ }^{5}$. O T. cruzi já foi objeto de pesquisa experimental em bioterapia para o câncer ${ }^{22}$

Estas observações indicam que múltiplos mecanismos estão envolvidos na inibição do processo de carcinogênese na colonopatia chagásica, com destaque para o processo de denervação mioentérica e seu papel na homeostase da mucosa intestinal. Investigações adicionais sobre a função destes componentes, aliadas a uma maior compreensão da biologia do T. cruzie da interação parasitohospedeiro, devem certamente elucidar a complexa associação entre a doença de Chagas e seu efeito protetor sobre a neoplasia colorretal.

\title{
A B S T R A C T
}

\begin{abstract}
The authors analyze the relation between gastrointestinal carcinogenesis and Chagas disease, based on detailed review of the literature. To this end, epidemiological, experimental and human material pathology description studies have been selected. The article discusses the possibility of protection being afforded by not fully known morphokinetic cellular, immune and neuroendocrine factors that would be secondary to plexus degeneration. Also aspects related to the parasite-host interaction from the viewpoint of epithelial modulation of colonic mucosa and its antitumor implications are presented. Finally, it exposes the pathophysiological mechanism of esophageal cancer development in patients with mega-organ. In conclusion, chagasic colopathy, especially the intrinsic neuronal damage, is a study model that can contribute to the understanding of colorectal carcinogenesis.
\end{abstract}

Key words: Chagas disease. Autonomic denervation. Colorectal neoplasms. Megaesophagus. Esophageal neoplasms.

\section{REFERENNCIAS}

1. Pajecki D, Zilberstein B, Santos MAA, Quintanilha AG, Cecconello I, Gama-Rodrigues J. Microbiota do megaesôfago e carcinogênese. Arq Gastroenterol. 2003;40(1):16-9.

2. Garcia SB, Oliveira JSM, Pinto LZ, Muccillo G, Zucoloto S. The relationship between megacolon and carcinoma of the colon: an experimental approach. Carcinogenesis. 1996;17(8):1777-9.

3. Adad SJ, Etchebehere RM, Araújo JR, Madureira AB, Lima VGF, Silva $A A$ et al. Association of chagasic megacolon and cancer of the colon: case report and review of the literature. Rev Soc Bras Med Trop. 2002;35(1):63-8.

4. Oliveira EC, Leite MSB, Miranda JA, Andrade AL, Garcia SB, Luquetti AO, Moreira H. Chronic Trypanosoma cruzi infection associated with low incidence of 1,2-dimethylhydrazine-induced colon cancer in rats. Carcinogenesis. 2001;22(5):737-40.

5. Garcia SB, Paiva A, Basile F, Aranha A, Zucoloto S. A retrospective study of histopathological findings in 894 cases of megacolon. What is the relationship between megacolon and colonic cancer? Rev Inst Med Trop S Paulo. 2003;45(2):91-3.
6. Polli-Lopes AC, Zucoloto S, Cunha FQ, Figueiredo LAS, Garcia SB. Myenteric denervation reduces the incidence of gastric tumors in rats. Cancer Lett. 2003;190(1):45-50.

7. Hoffman $\mathrm{HH}$, Schnitzlein HN. The number of nerve fibers in the vagus nerve of man. Anat Rec. 1961;139:429-35.

8. Gershon MD, Kirchgessner AL, Wade PR. Functional anatomy of the enteric nervous system. In: Johnson LR, editor. Physiology of the gastrointestinal tract. New York, Raven Press. 1994;381-422.

9. Furness $J B$, Costa $M$. Types of nerves in the enteric nervous system. Neuroscience. 1980;5(1):1-20

10. Genton L, Kudsk KA. Interactions between the enteric nervous system and the immune system: role of neuropeptides and nutrition. Am J Surg. 2003;186(3):253-8.

11. Ribeiro Júnior U, Safatle-Ribeiro AV, Habr-Gama A, Gama-Rodrigues JJ, Sohn J, Reynolds JC. Análise das alteraçöes do óxido nítrico em pacientes com megacólon chagásico. Rev Bras Colo-Proctol. 1998;18(1):52-7.

12. Marino AP, Silva AA, Pinho RT, Lannes-Vieira J. Trypanosoma cruzi infection: a continuous invader-host cell cross talk with participation of extracellular matrix and adhesion and chemoattractant molecules. Braz J Med Biol Res. 2003;36(8):1121-33. 
13. Iantorno G, Bassotti G, Kogan Z, Lumi CM, Cabanne AM, Fisogni S et al. The enteric nervous system in chagasic and idiopathic megacolon. Am J Surg Pathol. 2007;31(3):460-8.

14. Sanchez-Lermen RLP, Dick E, Salas JAP, Fontes CJF. Sintomas do trato digestivo superior e distúrbios motores do esôfago em pacientes portadores da forma indeterminada da doença de Chagas crônica. Rev Soc Bras Med Trop. 2007;40(2):197-203.

15. Vespúcio MVO, Turatti A, Modiano P, Oliveira EC, Chicote SRM, Pinto AMP et al. Intrinsic denervation of the colon is associated with a decrease of some colonic preneoplastic markers in rats treated with a chemical carcinogen. Braz J Med Biol Res. 2008;41(4):311-7.

16. Escalante RD, Oliveira EC, Cunha FQ, Vespúcio MVO, Ribeiro-Silva A, Aprilli F et al. Trypanosoma cruzi infection and/or administration of the nonsteroidal anti-inflammatory nimesulide increase the number of colonic crypts overexpressing metallothioneins in rat colon carcinogenesis. Braz J Med Biol Res. 2006;39(7):895-9.

17. Estofolete CF, Botelho-Machado C, Taboga SR, Zucoloto S, PolliLopes AC, Gil CD. Effects of myenteric denervation on extracellular matrix fibers and mast cell distribution in normal stomach and gastric lesions. Cancer Cell Int. 2010;10:18.

18. Lawrence T. Inflammation and cancer: a failure of resolution? Trends Pharmacol Sci. 2007;28(4):162-5.

19. Clevers $H$. At the crossroads of inflammation and cancer. Cell. 2004;118(6):671-4.

20. Farrow B, Sugiyama Y, Chen A, Uffort E, Nealon W, Mark Evers B. Inflammatory mechanisms contributing to pancreatic cancer development. Ann Surg. 2004;239(6):763-9.

21. Vasto S, Carruba G, Lio D, Colonna-Romano G, Di Bona D, Candore $G$ et al. Inflammation, ageing and cancer. Mech Ageing Dev. $2009 ; 130(1-2): 40-5$.
22. Fagundes JJ, Góes JRN, Coy CSR, Ayrizono MLS, Mochizuki M, Chadu $\mathrm{M}$ et al. Associação entre megacólon chagásico e câncer do intestino grosso: apresentação de casos e revisão da literatura/ The association between chagasic megacolon and colorectal cancer: presentation of cases and revision of literature. Rev Bras Colo-Proctol. 2002;22(4):252-6.

23. Henry MACA, Lerco MM, Oliveira WK. Câncer do esôfago em paciente com megaesôfago chagásico. Arq Gastroenterol. 2007:44(2):151-5.

24. Hibbs JB Jr, Lambert LH Jr, Remington JS. Resistance to murine tumors conferred by chronic infection with intracellular protozoa, Toxoplasma gondii and Besnoitia jellisoni. J Infect Dis. 1971;124(6):587-92.

Recebido em 07/12/2010

Aceito para publicação em 20/01/2011

Conflito de interesse: nenhum

Fonte de financiamento: nenhum

\section{Como citar este artigo:}

Gullo CE, Estofolete CF, Gil CD, Christiano AB, Netinho JG. Formas digestivas da doença de Chagas e carcinogênese: um estudo de associação. Rev Col Bras Cir. [periódico na Internet] 2012; 39(2). Disponível em URL: http://www.scielo.br/rcbc

Endereço para correspondência:

João Gomes Netinho

Email: jgnetinho@riopreto.com.br 